\title{
Pleiotropic predisposition to Alzheimer's disease and educational attainment: insights from the summary statistics analysis
}

\author{
Alexander M. Kulminski (iD) Elena Loiko • \\ Yury Loika $\cdot$ Irina Culminskaya
}

Received: 6 September 2021 / Accepted: 28 October 2021 / Published online: 6 November 2021

(C) The Author(s), under exclusive licence to American Aging Association 2021

\begin{abstract}
Epidemiological studies report beneficial associations of higher educational attainment (EDU) with Alzheimer's disease (AD). Prior genome-wide association studies (GWAS) also reported variants associated with AD and EDU separately. The analysis of pleiotropic associations with these phenotypes may shed light on EDU-related protection against AD. We performed pleiotropic meta-analyses using Fisher's method and omnibus test applied to summary statistics for single nucleotide polymorphisms (SNPs) associated with $\mathrm{AD}$ and EDU in large-scale univariate GWAS at suggestive-effect $\left(5 \times 10^{-8}<p<0.1\right)$ and genome-wide $\left(p \leq 5 \times 10^{-8}\right)$ significance levels. We report 53 SNPs that attained $p \leq 5 \times 10^{-8}$ at least in one of the pleiotropic meta-analyses and were reported in the univariate GWAS at $5 \times 10^{-8}<p<$ 0.1. Of them, there were 46 pleiotropic SNPs according to Fisher's method. Additionally, Fisher's method identified 25 of 206 SNPs with pleiotropic effects, which attained $p \leq 5 \times 10^{-8}$ in the univariate GWAS. We showed that a large fraction of the pleiotropic
\end{abstract}

Supplementary Information The online version contains supplementary material available at https://doi. org/10.1007/s11357-021-00484-1.

A. M. Kulminski $(\bowtie) \cdot$ E. Loiko · Y. Loika ·

I. Culminskaya

Biodemography of Aging Research Unit, Social

Science Research Institute, Duke University, Durham,

NC 27708-0408, USA

e-mail: kulminsk@duke.edu associations was affected by a counterintuitive phenomenon of antagonistic genetic heterogeneity, which explains the increase, rather than decrease, of the significance of the pleiotropic associations in the omnibus test. Functional enrichment analysis showed that apart from cancers, gene set harboring the nonpleiotropic SNPs was characterized by late-onset AD and neurodevelopmental disorders. The pleiotropic gene set was characterized by a broad spectrum of progressive neurological and neuromuscular diseases and immune-mediated conditions, including progressive motor neuropathy, multiple sclerosis, Parkinson's disease, and severe AD. Our results suggest that disentangling genes harboring variants with and without pleiotropic associations with AD and EDU is promising for dissecting heterogeneity in biological mechanisms of AD.

Keywords Alzheimer's disease - Education · Pleiotropy $\cdot$ Heterogeneity $\cdot$ Complex traits $\cdot$ Aging

\section{Background}

Alzheimer's disease (AD) is an age-related neurodegenerative disorder, which prevalence and incidence dramatically increase with age [1]. Studies also report no change or even declining trend of prevalence and incidence in developed countries during recent decades owing to, in part, higher educational attainment (EDU) and the progress in controlling cardiovascular 
risk factors [2, 3]. The burden of $\mathrm{AD}$, however, is projected to increase over time due to population aging and potential adverse trends of AD risk factors such as obesity and diabetes [4, 5]. This epidemiological evidence suggests that interventions aiming to improve the protective effect of $\mathrm{AD}$ modifiable risk factors might be a promising strategy in AD prevention and reducing its burden [6].

The association of higher EDU (i.e., more years of schooling) with a lower risk of $\mathrm{AD}$ is among the most robust findings in $\mathrm{AD}$ research [6, 7]. Despite that, the nature of connections between EDU and AD (i.e., causal, correlative, confounding) remains poorly understood. One hypothesis is that EDU helps build and maintain the cognitive reserve, and, therefore, education is considered a proxy of cognitive reserve [8]. This hypothesis leverages the observations that age-related brain damage does not seem to be directly linked to the clinical manifestation of the disease [9-11]. People with a greater cognitive reserve may better tolerate pathological changes due to preexisting connections between neurons ensuring higher cognitive processing and compensatory adaptation and creating new connections [12]. Accordingly, the cognitive reserve can be a plausible mechanism of cognitive resilience [13].

The cognitive reserve hypothesis suggests the existence of biological mechanisms, which can create stronger connections between the neurons and ascertaining synaptic plasticity. If EDU can affect AD development through a cognitive reserve or any other education-related mechanism(s), there should be a common genetic basis of EDU and AD. For example, this basis can be represented by pleiotropic genes, which can shed light on a mechanism involved in the protection of EDU against AD development.

Because $\mathrm{AD}$ is a heterogeneous condition, the cognitive reserve does not exhaust all potential factors associated with its pathogenesis. For example, emerging research suggests that overall health and agingrelated processes quantified using a frailty index approach [14] may be important factors contributing to the relationship between AD pathology and cognition both as modulators and in an independent manner $[15,16]$. These latent factors may partly explain accumulating observations of discrepancies in correlations between $\mathrm{AD}$ manifestation, $\mathrm{AD}$ biomarkers, and neuropathological changes [16]. Overall, it is increasingly recognized that $\mathrm{AD}$ should be considered a condition determined by multiple, likely interacting, pathophysiological processes.

The view on $\mathrm{AD}$ as "a multiply determined condition" [16] warrants exploring new approaches in the analyses of genetic predisposition to $\mathrm{AD}$, particularly in the case of pleiotropy, herein referenced to as pleiotropic predisposition. These approaches should recognize the complex roles of genetic factors in heterogeneous, age-related traits such as AD in both univariate and pleiotropic contexts. This recognition can be beneficial in identifying conventional pleiotropic associations with age-related traits and unconventional ones, such as antagonistic genetic heterogeneity characterized by discrepancies between the associations of a genetic variant with different traits and correlation between them $[17,18]$.

Here, we leveraged a pleiotropic meta-analysis using Fisher's method and omnibus test [18] applied to publicly available summary statistics (effect sizes, standard errors, and $p$ values) for single nucleotide polymorphisms (SNPs) from large-scale univariate GWAS of AD [19] and EDU [20]. We examined pleiotropic predisposition to these outcomes and evaluated functional roles of gene sets harboring pleiotropic and non-pleiotropic SNPs.

\section{Methods}

Publicly available summary statistics

Summary statistics for AD [19] and EDU [20] were provided for 7,055,881 and 8,146,840 SNPs, respectively, whose minor allele frequency (MAF) was larger than $1 \%$. There were $6,814,698$ overlapping SNPs between these GWAS. After excluding 695 multi-allelic and palindromic SNPs, the analyzed set was reduced to $6,814,003$ SNPs.

Further analyses focused on SNPs, which attained and did not attain the genome-wide (GW) significance $\left(p<5 \times 10^{-8}\right)$ separately in the published GWAS. The GW significant set included 957 SNPs in 42 loci from the AD GWAS and 4,747 SNPs in 185 loci from the EDU GWAS, totaling 5,704 SNPs. There were no SNPs that attained GW significance in both univariate GWAS simultaneously. The set of SNPs that did not achieve GW significance in either univariate GWAS included 6,808,299 SNPs. 
Pleiotropic meta-analysis

We used two types of tests to perform a pleiotropic meta-analysis of AD and EDU. The first test used Fisher's method [21]. It combines $p$ values across phenotypes, assuming that the combining statistics are independent. Although this test is practical, its disadvantage is that it neither considers the directions of the associations with outcomes to be combined nor the potential correlation between them.

To examine the potential roles of these two factors, we used a more comprehensive omnibus test [22-24]. In this study, we have summary statistics from univariate GWAS for two outcomes $(K=2), \mathrm{AD}$ and EDU, for each SNP to test for pleiotropy. Then, the omnibus test in this two-dimensional case for an SNP $j$ reads (index $j$ is omitted):

$\hat{z}^{\prime} \boldsymbol{\Sigma}^{-1} \hat{z}=\left[\left(\hat{z}_{1}^{2} \Sigma_{22}-\hat{z}_{1} \hat{z}_{2} \Sigma_{21}\right)+\left(\hat{z}_{2}^{2} \Sigma_{11}-\hat{z}_{1} \hat{z}_{2} \Sigma_{12}\right)\right] / \operatorname{det}(\boldsymbol{\Sigma})$.

Here $\hat{z}$ is a $z$-score vector of associations of an SNP with outcomes $i \in(\overline{1, K})$ and $\boldsymbol{\Sigma}$ is the correlation matrix of the $z$-scores or outcomes [23, 24]. The $z$-score for the associations with $\mathrm{AD}$ was defined as $\hat{z}=\beta / \sigma$, where $\beta$ is the estimated effect size and $\sigma$ is the corresponding standard error of an association of a SNP with AD. GWAS of EDU [20] reported truncated estimates of the effect sizes and standard errors. More accurate estimation of the $z$-score for EDU was evaluated using the reported $p$ values given the sign of the corresponding effect size.

Under the null hypothesis $\left(\boldsymbol{\beta}_{\boldsymbol{i}}=\mathbf{0}\right)$, the test statistic follows a chi-square distribution with $K$ degrees of freedom:

$\hat{z}^{\prime} \Sigma^{-1} \hat{z} \sim \chi_{K}^{2}$,

from which we obtained a combined $p$ value for this pleiotropic test.

As follows from Equation (1) above, the correlation between phenotypes may not necessary attenuate the significance of the pleiotropic associations in the case when $\hat{z}_{1} \hat{z}_{2} \Sigma_{21}, \hat{z}_{1} \hat{z}_{2} \Sigma_{12}>0$, but also strengthen them when $\hat{z}_{1} \hat{z}_{2} \Sigma_{21}, \hat{z}_{1} \hat{z}_{2} \Sigma_{12}<0$. The latter implies a counterintuitive phenomenon of antagonistic genetic heterogeneity, which is common in the genetics of heterogeneous traits $[17,18]$. The increase of significance (i.e., a decrease of $p$ value) is due to a mismatch of the signs of the associations with complex outcomes (i.e., the sign of the product $\hat{z}_{1} \hat{z}_{2}$ ) and correlation between them (i.e., $\Sigma_{21}, \Sigma_{12}$ ).

\section{Correlation}

Correlation matrix $\boldsymbol{\Sigma}$ was constructed [24] by evaluating Pearson correlation coefficients between the AD and EDU phenotypes available in the Framingham Heart Study (FHS) parental and offspring cohorts [25], Cardiovascular Health Study (CHS) [26], and the National Institute on Aging (NIA) Late-Onset Alzheimer Disease Family Study (LOADFS) [27]. $\mathrm{AD}$ was categorized as 1 and 0 for the AD-affected and unaffected subjects, respectively. EDU variable was coded as years of education. The correlation coefficients between these phenotypes were evaluated in each cohort separately, $r=-0.03$ in CHS, $r=$ -0.03 in FHS, and $r=-0.31$ in LOADFS. The large magnitude of $r$ in the LOADFS is likely because of the AD case-control design of this study, which is different from the population/community-based longitudinal designs of the FHS and CHS cohorts. We also evaluated Pearson correlation coefficient between $\mathrm{AD}$ and EDU using summary statistics from the published AD and EDU GWAS, $r=-0.03$. Given these estimates, we constructed two 2-by-2 correlation matrices $\boldsymbol{\Sigma}$ with off-diagonal values $r=-0.03$ and $r$ $=-0.30$.

\section{Pleiotropic associations}

Fisher's method and the omnibus test can both be used to identify pleiotropic associations. An advantage of the omnibus test is that it takes into account correlation between phenotypes (see above). Correlation can be a sensitive parameter in the omnibus test $[17,18]$ and its accurate evaluation is desirable. This evaluation requires more detailed (individual- or study-level) data than those available from the published summary statistics. Because Fisher's method does not rely on correlation, it was our primary tool to define the pleiotropic effects. Using this method, the pleiotropic effect can be naturally defined when the pleiotropic $p$ value from Fisher's method is smaller than the $p$ value from either AD or EDU univariate GWAS. This definition has a clear interpretation because Fisher's method provides the probability of an event. When Fisher's $p$ value from a pleiotropic 
analysis is smaller than $p$ values from univariate analyses, this implies that the probability of the pleiotropic association with multiple phenotypes is larger than those of each of these phenotypes separately.

Fisher's method and the omnibus test penalize the estimates of $p$ values for testing multiple phenotypes by increasing the number of degrees of freedom for $\chi^{2}$. Therefore, the traditional GW level of significance, $p=5 \times 10^{-8}$, remains relevant and it was used for reporting GW significance attained in at least one pleiotropic meta-analysis using Fisher's method and/ or omnibus test.

\section{Index SNPs}

We selected one index SNP per gene or gene cluster if genes overlap. The index SNP was defined as an SNP with the smallest $p$ value attained in an omnibus test. We reported SNPs, which might be in the same locus (distinct loci are determined if they are separated by more than $1 \mathrm{M}$ base pairs or they are on different chromosomes), but were mapped to different genes because associations with AD and EDU may not be due to the same genes.

Gene mapping and functional enrichment analysis

SNPs were mapped to genes using variant effect predictor from Ensembl and the NCBI SNP database (assembly GRCh37.p13). For SNPs that were not in protein-coding regions, we selected the closest protein-coding gene(s). The vast majority of genes were within $\pm 100 \mathrm{~K}$ base pairs from the index SNP. Functional enrichment analysis was performed using the core analysis function available in the Ingenuity Pathway Analysis (IPA) tool (www.qiagenbioinform atics.com) to gain insights into potential underlying biological mechanisms of the observed associations.

\section{Results}

Pleiotropic associations for SNPs, which did not attain GW significance in the univariate GWAS of $\mathrm{EDU}$ or $\mathrm{AD}$

First, we performed a pleiotropic meta-analysis of summary statistics for the associations, which did not attain GW significance with AD [19] or EDU [20]. This analysis identified 53 SNPs, which were associated with either AD or EDU at $5 \times 10^{-8}<$ $p<0.1$, but they attained GW significance $(p \leq 5$ $\left.\times 10^{-8}\right)$ in at least one pleiotropic test, i.e., Fisher's method and/or omnibus test (Table 1 and Supplementary Table S1).

Of these 53 SNPs, Fisher's method identified pleiotropic associations for 46 SNPs, for which Fisher's $p$ value was smaller than the $p$ value for the association with either AD or EDU (Table 1, column Plt). Pleiotropic GW significance in this test was achieved for 10 of 46 SNPs located on chromosomes 2, 6, 7, and 17. For 45 of 46 pleiotropic SNPs (except rs12006296), $p$ values in the univariate analysis attained nominal significance $(p<0.05)$. In contrast, for the remaining seven of 53 SNPs, the univariate association with either EDU or $\mathrm{AD}$ was of suggestive-effect significance $(0.05<p<0.1)$.

Table 1 shows that signs of the univariate associations of the same alleles with AD and EDU are opposite for two of 53 SNPs only, rs 17504614 and rs6952006. This is an intuitively expected relationship because it is consistent with the negative correlation between AD and EDU. Then, it is anticipated that the omnibus test penalizing for correlation between $\mathrm{AD}$ and EDU returns less significant estimates than Fisher's method, which, in this case, becomes below GW level, $p>5 \times 10^{-8}$.

The signs of the associations of the same alleles from the remaining 51 SNPs with EDU and AD are, however, the same. This relationship is counterintuitive because of the negative correlation between $\mathrm{AD}$ and EDU. The same directions of genetic associations with $\mathrm{AD}$ and EDU and negative correlation between them imply the case of antagonistic heterogeneity [18]. This counterintuitive heterogeneity leads to increasing the significance (i.e., smaller $p$ values), rather than decreasing, of the pleiotropic effects for the correlated traits in the omnibus test compared to Fisher's method. This increase is modest when the correlation is small (Table $1, r=$ -0.03 ), but it is substantially larger, ranging from 1.8 to 3.2 orders of magnitude when the correlation is larger (Table 1, $r=-0.30$ ). Antagonistic heterogeneity supports pleiotropic associations for the remaining seven of 53 SNPs, which were not selected as pleiotropic using Fisher's method. 
Table 1. Genome-wide significant pleiotropic associations for SNPs, which did not attain genome-wide significance in the univariate GWAS of education or Alzheimer's disease.

\begin{tabular}{|c|c|c|c|c|c|c|c|c|c|c|c|c|c|}
\hline \multicolumn{5}{|c|}{ Locus-SNP information } & \multicolumn{2}{|c|}{ Education } & \multicolumn{2}{|c|}{$\begin{array}{l}\text { Alzheimer's } \\
\text { disease }\end{array}$} & \multicolumn{5}{|c|}{ Pleiotropic analysis } \\
\hline SNP & $\mathrm{Chr}$ & $\mathrm{os}, \mathrm{kb}$ & $\mathrm{A} 1$ & EAF & $\beta_{\mathrm{EDU}}$ & $P_{\mathrm{EDU}}$ & $\beta_{\mathrm{AD}}$ & $P_{\mathrm{AD}}$ & $P_{\text {Fisher }}$ & $P_{\text {Omni1 }}$ & $P_{\text {Omni2 }}$ & Plt & $\mathrm{AH}$ \\
\hline 37436 & 1 & 94,039 & $\mathrm{~T}$ & 0.23 & 0.015 & 3.37E-07 & -0.050 & $8.56 \mathrm{E}-03$ & $5.95 \mathrm{E}-08$ & 4.63E-08 & $1.66 \mathrm{E}-10$ & Yes & Yes \\
\hline 477432 & 1 & 112,185 & A & 0.57 & 012 & $4 \mathrm{E}-06$ & 34 & 3.99E-02 & $8.12 \mathrm{E}-07$ & $6.38 \mathrm{E}-07$ & $2 \mathrm{E}-09$ & Yes & Yes \\
\hline 113830567 & 1 & 207,677 & $\mathrm{~T}$ & 0.20 & 006 & 7.03E-02 & 0.099 & $1.34 \mathrm{E}-06$ & $1.61 \mathrm{E}-06$ & $1.24 \mathrm{E}-06$ & $2.44 \mathrm{E}-08$ & No & Yes \\
\hline rs 17504614 & 2 & 51,080 & $\mathrm{~T}$ & 0.82 & 0.015 & $6.37 \mathrm{E}-07$ & -0.063 & $2.78 \mathrm{E}-03$ & $3.74 \mathrm{E}-08$ & 7.23E-08 & $1.20 \mathrm{E}-06$ & Yes & No \\
\hline 268134 & 2 & 65,608 & A & 0.24 & 015 & $.91 \mathrm{E}-07$ & 0.048 & $6.65 \mathrm{E}-03$ & $2.73 \mathrm{E}-08$ & $2.09 \mathrm{E}-08$ & $58 \mathrm{E}-11$ & Yes & Yes \\
\hline 10172113 & 2 & 226,618 & $\mathrm{~T}$ & 0.25 & -0.014 & $6.30 \mathrm{E}-07$ & -0.044 & $1.31 \mathrm{E}-02$ & $1.62 \mathrm{E}-07$ & $1.28 \mathrm{E}-07$ & $6.89 \mathrm{E}-10$ & Yes & Yes \\
\hline 003901 & 3 & 25,1 & A & 0.18 & 5 & & & & & $9.50 \mathrm{E}-07$ & $4 \mathrm{E}-09$ & Yes & Yes \\
\hline 17785248 & 3 & 47,6 & A & 0.75 & 0.014 & 1.28 & 0.031 & 8.79E-02 & 1.911 & $1.45 \mathrm{E}-06$ & E-08 & No & Yes \\
\hline 1665982 & 3 & 47,905 & A & 0.27 & -0.014 & $4.25 \mathrm{E}-07$ & & $3.24 \mathrm{I}$ & $2.63 \mathrm{E}-07$ & $2.02 \mathrm{E}-07$ & E-09 & Yes & Yes \\
\hline & 3 & 49, & $\mathrm{~T}$ & 0.04 & 032 & $1.22 \mathrm{E}-06$ & 01 & 3.19 & 7.02E-07 & $5.56 \mathrm{E}-07$ & -09 & Yes & Yes \\
\hline 10446472 & 3 & 51,294 & A & 0.16 & -0.017 & $1.40 \mathrm{E}-06$ & -0.082 & $4.52 \mathrm{E}-02$ & $1.11 \mathrm{E}-06$ & $8.78 \mathrm{E}-07$ & 7E-08 & Yes & Yes \\
\hline 09 & 4 & 1,7 & A & 0. & 0 & 2. & 0. & 3.2 & 1.6 & 1.3 & 08 & es & Yes \\
\hline 82 & 4 & 66,2 & $\mathrm{~T}$ & 0.98 & -0.0 & $10 \mathrm{E}-04$ & 7 & $1.01 \mathrm{E}-03$ & $1.90 \mathrm{E}-06$ & $1.72 \mathrm{E}-06$ & E-08 & Yes & Yes \\
\hline 82 & 5 & 62 & A & 0.43 & 0.012 & $3.88 \mathrm{E}-06$ & 0.1 & 2.09E-02 & $1.41 \mathrm{E}-06$ & $1.17 \mathrm{E}-06$ & E-08 & Yes & Yes \\
\hline 615 & 6 & & $\mathrm{~T}$ & 0.9 & 3 & 06 & & & & -06 & & No & Yes \\
\hline 18 & 6 & 32 , & A & 0.86 & -0.013 & $1.59 \mathrm{E}-04$ & -0 . & $9.63 \mathrm{E}-04$ & $2.56 \mathrm{E}-06$ & $2.34 \mathrm{E}-06$ & -08 & Yes & Yes \\
\hline 140677956 & 6 & 32,340 & $\mathrm{~T}$ & 0.45 & -0.012 & $6.65 \mathrm{E}-05$ & -0 & $2.40 \mathrm{E}-04$ & $3.02 \mathrm{E}-07$ & $2.63 \mathrm{E}-07$ & E-10 & Yes & Yes \\
\hline 261 & 6 & 32,3 & $\mathrm{~T}$ & 0.7 & -0.008 & $9.54 \mathrm{E}-03$ & & -08 & $1.70 \mathrm{E}-08$ & E-08 & E-11 & Yes & Yes \\
\hline 00 & 6 & 32,410 & $\mathrm{~T}$ & 0.10 & 0.013 & $3.50 \mathrm{E}-03$ & 0.106 & $3.89 \mathrm{E}-05$ & $2.28 \mathrm{E}-06$ & $2.05 \mathrm{E}-06$ & E-08 & Yes & Yes \\
\hline 7200 & 6 & & $\mathrm{~T}$ & 0. & 0.01 & & & & & & & es & Yes \\
\hline 15 & 6 & 32 & A & 0. & -0.013 & $1.25 \mathrm{E}-05$ & -0 & $6.77 \mathrm{E}-05$ & $1.85 \mathrm{E}-08$ & 1.49E-08 & $8-11$ & Yes & Yes \\
\hline 26 & 6 & 32,6 & A & 0.71 & -0 . & 5.0 & -0 & 1.4 & 1.5 & 08 & 11 & Yes & Yes \\
\hline 5/501 & 6 & & $\pi$ & & & & & & & & & o & Yes \\
\hline 1761608 & 6 & 109 & A & 0.41 & 011 & 4.8 & 0 & 03 & $1.23 \mathrm{E}-07$ & 1.02E-07 & -10 & Yes & Yes \\
\hline rs1533827 & 7 & 2,0 & $\mathrm{~T}$ & & & & & & & & & es & Yes \\
\hline & 7 & 8 , & $\mathrm{T}$ & & & & & & & 08 & & Yes & No \\
\hline 79 & 7 & 114 & $\mathrm{~T}$ & 0.44 & -0.011 & 1.06 & -0 & 1.3 & 2.45 & -06 & 08 & Yes & Yes \\
\hline rs536332 & 8 & & A & & & & & & & & & es & Yes \\
\hline rs35927132 & 8 & 28, & $\mathrm{~T}$ & 0.1 & 0.014 & $7.85 \mathrm{E}-05$ & 0.0 & $2.08 \mathrm{E}-03$ & $2.72 \mathrm{E}-06$ & $2.47 \mathrm{E}-06$ & E-08 & Yes & Yes \\
\hline rs12675931 & 8 & & $\mathrm{~T}$ & 0.7 & 0.011 & 1.8 & & & 6.1 & $5.46 \mathrm{E}-07$ & -09 & Yes & Yes \\
\hline rs 2631024 & 8 & 91,996 & A & 0.2 & -0.010 & 4.72E-04 & & 3.50E-04 & $2.74 \mathrm{E}-06$ & $2.52 \mathrm{E}-06$ & -08 & Yes & Yes \\
\hline 066296 & 9 & 3,142 & $\mathrm{C}$ & 0.34 & 0.012 & $2.65 \mathrm{E}-06$ & 0.031 & 5.39E-02 & 2.3 & $1.91 \mathrm{E}-06$ & -08 & Yes & Yes \\
\hline rs7912495 & 10 & 11,719 & A & 0.53 & -0.008 & $1.96 \mathrm{E}-03$ & -0.063 & $1.07 \mathrm{E}-04$ & $3.44 \mathrm{E}-06$ & $3.15 \mathrm{E}-06$ & $2.59 \mathrm{E}-08$ & Yes & Yes \\
\hline rs12219346 & 10 & & $\mathrm{~T}$ & & & $9.55 \mathrm{E}-08$ & & 4.93 & $9.50 \mathrm{E}-08$ & $6.87 \mathrm{E}-08$ & $6.09 \mathrm{E}-10$ & Yes & Yes \\
\hline rs 4600211 & 11 & 24,725 & $\mathrm{~T}$ & 0.79 & -0.014 & $2.79 \mathrm{E}-06$ & -0.039 & $4.26 \mathrm{E}-02$ & $2.02 \mathrm{E}-06$ & $1.63 \mathrm{E}-06$ & $2.63 \mathrm{E}-08$ & Yes & Yes \\
\hline 6043 & 11 & 85, & $\mathrm{~T}$ & 0.9 & -0.019 & $3.67 \mathrm{E}-02$ & & 5.79E-06 & 3.48 & 2.89E-06 & $4.98 \mathrm{E}-08$ & Yes & Yes \\
\hline rs9509558 & 13 & & $\mathrm{~T}$ & 0.4 & -0.009 & $5.29 \mathrm{E}-04$ & & $5.16 \mathrm{E}-04$ & & 4.09E-06 & $3.42 \mathrm{E}-08$ & Yes & Yes \\
\hline rs45618332 & 14 & 64,906 & $\mathrm{~T}$ & 0.24 & 0.013 & 3.19E-06 & 0.040 & $2.58 \mathrm{E}-02$ & $1.42 \mathrm{E}-06$ & $1.17 \mathrm{E}-06$ & $1.41 \mathrm{E}-08$ & Yes & Yes \\
\hline 100349 & 14 & & A & 0.6 & -0.013 & -06 & & & & -07 & & No & Yes \\
\hline 7160634 & 14 & 65,001 & A & 0.33 & 0.013 & $9.27 \mathrm{E}-07$ & 0.028 & $8.52 \mathrm{E}-02$ & $1.37 \mathrm{E}-06$ & $1.03 \mathrm{E}-06$ & $2.18 \mathrm{E}-08$ & No & Yes \\
\hline 3098169 & 15 & 50 & A & 0.49 & -0.010 & $8.00 \mathrm{E}-05$ & -0 & $3.81 \mathrm{E}-03$ & $4.87 \mathrm{E}-06$ & $4.46 \mathrm{E}-06$ & $4.52 \mathrm{E}-08$ & Yes & Yes \\
\hline & 15 & & A & 0.2 & -0.0 & & & $2.98 \mathrm{E}-05$ & 4.41E-06 & $3.93 \mathrm{E}-06$ & 4.61E-08 & Yes & Yes \\
\hline 113294283 & 15 & 64,346 & A & 0.10 & 0.010 & $4.28 \mathrm{E}-02$ & 0.189 & 4.47E-06 & $3.15 \mathrm{E}-06$ & $2.58 \mathrm{E}-06$ & 4.64E-08 & Yes & Yes \\
\hline
\end{tabular}


Table 1. (continued)

\begin{tabular}{|c|c|c|c|c|c|c|c|c|c|c|c|c|c|}
\hline \multicolumn{5}{|c|}{ Locus-SNP information } & \multicolumn{2}{|c|}{ Education } & \multicolumn{2}{|c|}{$\begin{array}{l}\text { Alzheimer's } \\
\text { disease }\end{array}$} & \multicolumn{5}{|c|}{ Pleiotropic analysis } \\
\hline SNP & Chr & Pos, kb & A1 & EAF & $\beta_{\mathrm{EDU}}$ & $P_{\mathrm{EDU}}$ & $\beta_{\mathrm{AD}}$ & $P_{\mathrm{AD}}$ & $P_{\text {Fisher }}$ & $P_{\text {Omnil }}$ & $P_{\text {Omni2 }}$ & Plt & $\mathrm{AH}$ \\
\hline s116926649 & 17 & 43,507 & A & 0.23 & -0.017 & $4.46 \mathrm{E}-07$ & -0.036 & 7.94E-02 & $6.43 \mathrm{E}-07$ & 4.75E-07 & $8.26 \mathrm{E}-09$ & No & $\mathrm{Ye}$ \\
\hline rs143982995 & 17 & 43,5 & $\mathrm{~T}$ & 0.22 & -0.016 & $3.62 \mathrm{E}-06$ & -0.067 & $9.61 \mathrm{E}-03$ & $6.33 \mathrm{E}-07$ & $5.28 \mathrm{E}-07$ & $3.65 \mathrm{E}-09$ & Yes & Yes \\
\hline rs 150180355 & 17 & 43,687 & A & 0.25 & -0.015 & $8.15 \mathrm{E}-05$ & -0.091 & 2.39E-04 & $3.66 \mathrm{E}-07$ & $3.20 \mathrm{E}-07$ & $1.01 \mathrm{E}-09$ & Yes & Yes \\
\hline rs117086266 & 17 & 44,077 & A & 0.73 & 0.016 & 3.34E-07 & 0.046 & $1.82 \mathrm{E}-02$ & $1.21 \mathrm{E}-07$ & $9.36 \mathrm{E}-08$ & $5.38 \mathrm{E}-10$ & Yes & Yes \\
\hline rs143364530 & 17 & 44,132 & A & 0.28 & -0.017 & $1.16 \mathrm{E}-07$ & -0.048 & $1.38 \mathrm{E}-02$ & 3.41E-08 & $2.56 \mathrm{E}-08$ & $9.65 \mathrm{E}-11$ & Yes & Yes \\
\hline rs 199533 & 17 & 44,829 & A & 0.25 & -0.017 & $7.16 \mathrm{E}-08$ & -0.048 & $1.14 \mathrm{E}-02$ & $1.79 \mathrm{E}-08$ & $1.33 \mathrm{E}-08$ & $3.95 \mathrm{E}-11$ & Yes & Yes \\
\hline rs199503 & 17 & 44,862 & A & 0.25 & -0.017 & $5.86 \mathrm{E}-08$ & -0.049 & $1.18 \mathrm{E}-02$ & $1.52 \mathrm{E}-08$ & $1.12 \mathrm{E}-08$ & $3.25 \mathrm{E}-11$ & Yes & Yes \\
\hline rs12611358 & 19 & 46,251 & A & 0.42 & 0.007 & 4.65E-03 & 0.067 & $1.63 \mathrm{E}-05$ & $1.32 \mathrm{E}-06$ & $1.15 \mathrm{E}-06$ & 8.09E-09 & Yes & Yes \\
\hline rs2014576 & 19 & 46,269 & A & 0.44 & 0.006 & $1.69 \mathrm{E}-02$ & 0.071 & $8.68 \mathrm{E}-06$ & $2.45 \mathrm{E}-06$ & $2.09 \mathrm{E}-06$ & $2.48 \mathrm{E}-08$ & Yes & res \\
\hline rs2837992 & 21 & 42,621 & $\mathrm{~T}$ & 0.39 & 0.013 & $3.08 \mathrm{E}-07$ & 0.037 & $2.26 \mathrm{E}-02$ & $1.38 \mathrm{E}-07$ & $1.06 \mathrm{E}-07$ & $6.87 \mathrm{E}-10$ & Yes & Yes \\
\hline
\end{tabular}

Chr denotes chromosome; Pos, kb denotes position in kilo bases; EAF denotes frequency of the effect allele indicated in column A1

Subscripts EDU and AD indicate the estimates of the effect sizes $(\beta)$ and $p$ value $(P)$ from published genome-wide association studies of education and Alzheimer's disease, respectively.

Columns $\boldsymbol{P}_{\text {Fisher }}, \boldsymbol{P}_{\text {Omni1 }}$, and $\boldsymbol{P}_{\text {Omni2 }}$ show $p$ values from the pleiotropic analyses using Fisher's method and two omnibus tests, respectively. Subscripts Omni1 and Omni2 denote the results of the pleiotropic analyses using omnibus tests with Pearson correlation coefficients between Alzheimer's disease and education $r=-0.03$ and $r=-0.30$, respectively.

Column Plt indicates pleiotropy (yes) defined based on Fisher's method. Column AH indicates antagonistic pleiotropy (yes).

More details are given in Supplementary Table S1.

Pleiotropic associations for SNPs which attained GW significance with EDU or AD

We identified 169 SNPs (mapped to different genes) at GW level of significance in GWAS of EDU and 37 SNPs in GWAS of AD, totaling 206 GW significant associations (Supplementary Table S2). No SNPs attained GW significance in both univariate GWAS simultaneously. For the vast majority of these SNPs, 167 of 206, the associations reaching GW significance for one trait did not attain even suggestiveeffect significance $(p>0.1)$ for the other trait.

Fisher's method identified pleiotropic effects for 25 of 206 SNPs (Table 2). For all of them, the associations reaching GW significance for one trait also attained the nominal significance $(p<0.05)$ for the other trait. For eight of 25 SNPs, we also observed antagonistic heterogeneity. Such heterogeneity was seen for six other SNPs, which attained GW significance for one trait and at least suggestive-effect significance $(p<0.1)$ for the other trait (Supplementary Table S2). As antagonistic heterogeneity increases the significance, it supports pleiotropic associations for these six SNPs.
Functional enrichment analysis

Our analysis was performed for genes harboring pleiotropic and non-pleiotropic SNPs (herein referred to as pleiotropic and non-pleiotropic gene sets, respectively) separately. Pleiotropic SNPs were consistently defined based on Fisher's method (Tables 1 and 2 and Supplementary Table S2). Overall, 75 and 195 genes were selected for the pleiotropic and non-pleiotropic SNPs, respectively. We did not include NECTIN2, TOMM40, APOE, and $A P O C 1$ genes because AD GWAS reported $p$ values for SNPs selected in these genes as zero, and, therefore, pleiotropy could not be reliably identified using Fisher's method. We examined gene enrichment in pathways (defined by ingenuity canonical pathways) and categories of diseases and disorders and biological functions (defined by the ingenuity molecular and cellular functions and physiological system development and function), as discussed below. We did not examine enrichment if these biological terms were represented by one gene only. 
Table 2. SNPs attaining genome-wide significance in the univariate GWAS of education or Alzheimer's disease and showing pleiotropic associations with these outcomes.

\begin{tabular}{|c|c|c|c|c|c|c|c|c|c|c|c|c|c|}
\hline \multicolumn{5}{|c|}{ Locus-SNP information } & \multicolumn{2}{|c|}{ Education } & \multicolumn{2}{|c|}{$\begin{array}{l}\text { Alzheimer's } \\
\text { disease }\end{array}$} & \multicolumn{5}{|c|}{ Pleiotropic analysis } \\
\hline NP & $\mathrm{Chr}$ & b & A1 & EAF & U & 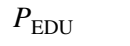 & $\beta_{1}$ & $P_{\mathrm{AD}}$ & 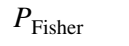 & $P_{\text {Omnil }}$ & $P_{\text {Omni2 }}$ & lt & \\
\hline 8 & 1 & & $\mathrm{~T}$ & 0. & 6 & 1 & & 2 & 69E-11 & $29 \mathrm{E}-11$ & 30 & es & \\
\hline & 2 & & 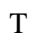 & & & & & & & & & Yes & \\
\hline 3172494 & 3 & & $\mathrm{~T}$ & & 206 & $90 \mathrm{E}-11$ & & & $62 \mathrm{E}-11$ & $89 \mathrm{E}-12$ & $\mathrm{e}-15$ & Yes & \\
\hline & 3 & & $\mathrm{~T}$ & 0 & 26 & & 0.0 & & $5 \mathrm{E}-09$ & -10 & -12 & Yes & \\
\hline 7709 & 5 & & A & & & & & & & & & Yes & \\
\hline 113 & 5 & 60 & $\mathrm{~T}$ & 0.12 & 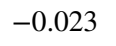 & & & & & 09 & & es & \\
\hline 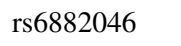 & 5 & & 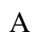 & & & & & & & & & es & \\
\hline 27732 & 5 & 87 , & A & 0.47 & 017 & $4.06 \mathrm{E}-11$ & -0 & $2.11 \mathrm{E}-02$ & 2.46 & $3.71 \mathrm{E}-11$ & 10 & Yes & \\
\hline 51 & 5 & & A & 0. & & & & & & & & es & \\
\hline 50651 & 5 & & $\mathrm{~T}$ & & S & & & & 1 & & 09 & Yes & \\
\hline 13 & 6 & & $\mathrm{~T}$ & & 011 & & & & & & & es & \\
\hline 77212 & 6 & & $T$ & & & & & & & & & es & \\
\hline 3890065 & 11 & & $\mathrm{C}$ & & 15 & 8 & & 3 & & 09 & 08 & Yes & \\
\hline . & 11 & 95 & $\mathrm{~T}$ & 0. & & & & & & & & res & \\
\hline & 11 & & $\mathrm{~T}$ & & & & & & & & 12 & Yes & \\
\hline 6 & 12 & 12 & $\mathrm{C}$ & 0 & -0.021 & 7.7 & 0 . & 2 & & 13 & & Yes & \\
\hline . & 12 & & $\mathrm{C}$ & & & & & & & & & Yes & \\
\hline $55 / 42290$ & 12 & & $\mathrm{~T}$ & & -0.019 & 2 & & 3 & 13 & 13 & 12 & Yes & \\
\hline & 12 & & A & & O & 5 & & & & & 3. & Yes & \\
\hline 10744150 & 12 & & A & & & 15 & 0 & 03 & -16 & 15 & e-14 & Yes & \\
\hline & 12 & & A & 0.73 & -0.023 & & & & & & & Yes & \\
\hline & 14 & & A & & & 3 & & & & 1.0 & E-07 & Yes & \\
\hline 141 & 17 & & A & & & & & & & & -11 & Yes & \\
\hline rs116926346 & 17 & 43,931 & A & 0.24 & -0.018 & 4.45E-08 & -0.050 & $1.27 \mathrm{E}-02$ & $1.26 \mathrm{E}-08$ & $9.24 \mathrm{E}-09$ & $2.64 \mathrm{E}-11$ & Yes & \\
\hline$s 113568679$ & 17 & 43,991 & A & 0.73 & 0.018 & $3.03 \mathrm{E}-08$ & 0.050 & $9.67 \mathrm{E}-03$ & 6.72E-09 & 4.87E-09 & $1.06 \mathrm{E}-11$ & Yes & \\
\hline
\end{tabular}

Chr denotes chromosome; Pos, kb denotes position in kilo bases; EAF denotes frequency of the effect allele indicated in column A1

Subscripts EDU and AD indicate the estimates of the effect sizes $(\beta)$ and $p$ value $(P)$ from published genome-wide association studies of education and Alzheimer's disease, respectively.

Columns $\boldsymbol{P}_{\text {Fisher }}, \boldsymbol{P}_{\text {Omni1 }}$, and $\boldsymbol{P}_{\text {Omni2 }}$ show $p$ values from the pleiotropic analyses using Fisher method and two omnibus tests, respectively. Subscripts Omni1 and Omni2 denote the results of the pleiotropic analyses using omnibus tests with Pearson correlation coefficients between Alzheimer's disease and education $r=-0.03$ and $r=-0.30$, respectively.

Column Plt indicates pleiotropy (yes) defined based on Fisher's method. Column AH indicates antagonistic pleiotropy (yes).

More details are given in Supplementary Table S2.

\section{Pathways}

Pathway analysis of the pleiotropic gene set identified 29 separate, but having overlapping genes, ingenuity canonical pathways, which were significant at the false discovery rate (FDR) $p_{\mathrm{FDR}}<0.05$ (i.e., after adjustment for multiple testing [28]) (Supplementary Table S3). Twenty-eight of them were characterized by enrichment of human leukocyte antigen (HLA) genes and were mainly immune and inflammatory pathways. The remaining synaptogenesis signaling pathway is involved in synapse formation, function, and maintenance. None of the pathways were significant at $p_{\mathrm{FDR}}<0.05$ for the set of 195 non-pleiotropic genes. 


\section{Diseases and disorders}

The top five significant IPA disease and disorder categories for the non-pleiotropic gene set included cancer, gastrointestinal disease, organismal injury and abnormalities, dermatological diseases and conditions, and neurological disease (Figure 1A). Four of them, except dermatological diseases and conditions, were in the top five for the pleiotropic gene set, but with the neurological disease on the top, followed by skeletal and muscular disorders, and then by cancer, organismal injury and abnormalities, and gastrointestinal disease (Figure 1B).

Disease annotation showed enrichment of cancers at different sites (with the prevalence of extracranial solid tumor and digestive organ tumor) in both nonpleiotropic and pleiotropic gene sets, although this enrichment was more pronounced in the non-pleiotropic gene set (Supplementary Tables S4 and S5). Apart from cancers, both pleiotropic and non-pleiotropic sets were enriched for the same three brain disorders (familial AD, familial encephalopathy, familial schizophrenia), hypoplasia, and inflammatory bowel diseases, specifically Crohn's disease (Table 3).

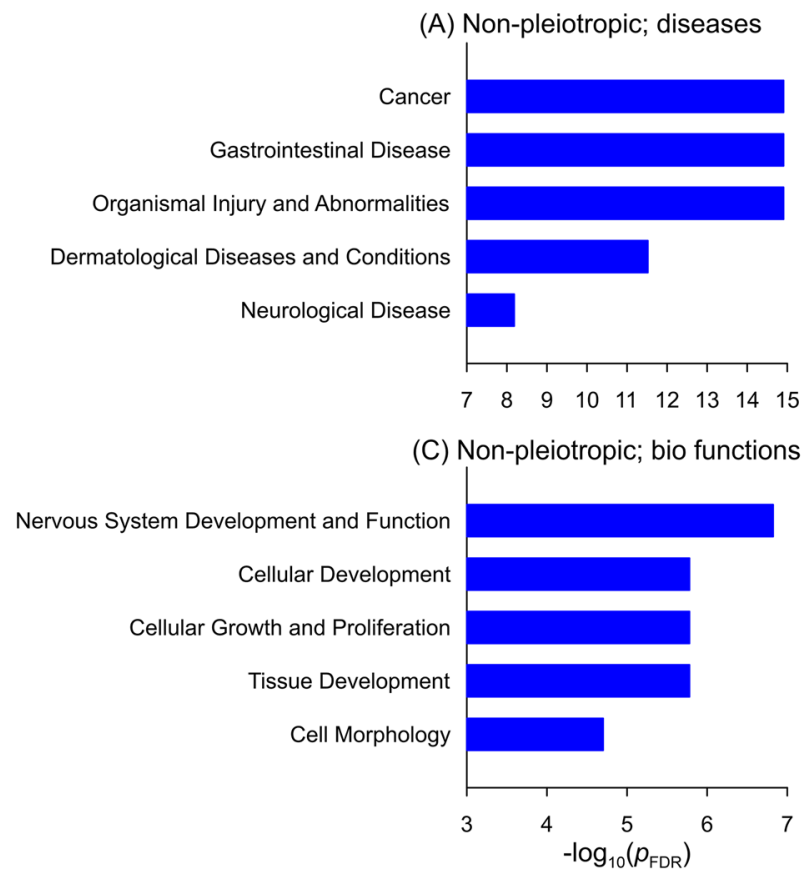

Fig. 1. Top five categories of diseases and disorders (A and B) and biological functions ( $\mathbf{C}$ and $\mathbf{D})$ enriched in the nonpleiotropic (A and $\mathbf{C}$ ) and pleiotropic (B and $\mathbf{D})$ gene sets. The
Annotation of non-cancer diseases and disorders identified 38 sub-categories enriched $\left(p_{\mathrm{FDR}}<0.05\right)$ only in the non-pleiotropic gene set (Table 4 and Supplementary Table S4). Brain disorders, including lateonset $\mathrm{AD}$, were at the top. In addition, we observed enrichment of neurodevelopmental disorders, such as congenital malformation of the brain, autism spectrum disorder (ASD), and mental retardation, which can adversely affect cognitive function and academic performance. The non-pleiotropic gene set was also enriched for conditions related to abnormal organ development (e.g., dysgenesis, aplasia, or hypoplasia) and several specific disorders (e.g., amyloidosis, microangiopathy, lesioning of the aorta, early-onset obesity).

In the pleiotropic gene set, we identified 67 disease and disorder sub-categories with enrichment at $p_{\text {FDR }}<0.05$ (Table 4 and Supplementary Table S5). Top conditions were related to progressive neurological and neuromuscular diseases and movement disorders, including progressive motor neuropathy $(\mathrm{PMN})$, multiple sclerosis (MS), progressive parkinsonism, Creutzfeldt-Jakob disease, and severe AD, which are often immune-mediated. Also, this set

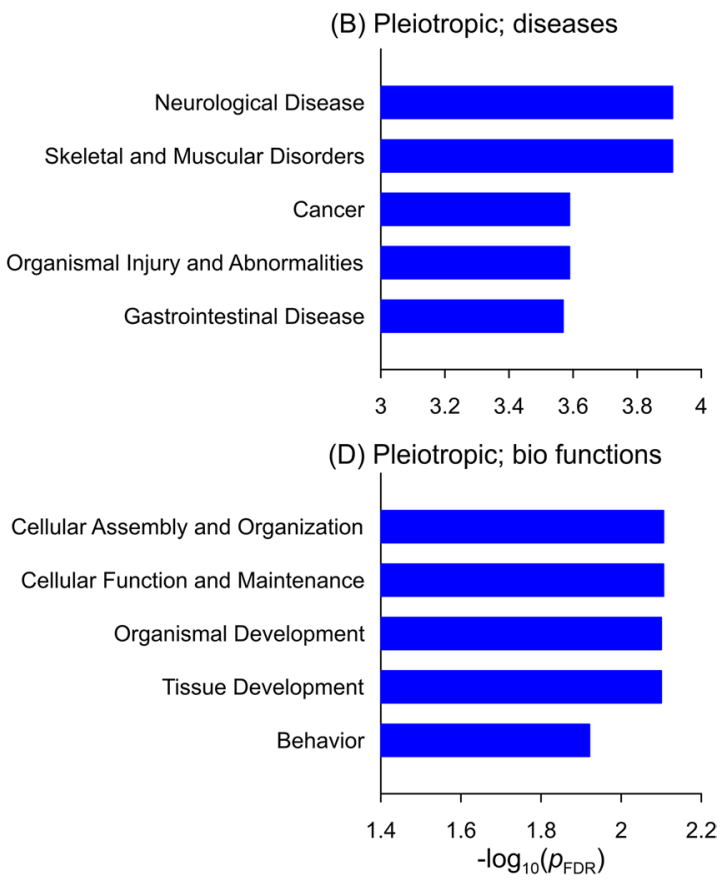

$x$-axis shows minus log-base-10 transformed false discovery rate adjusted $p$ value $\left(p_{\mathrm{FDR}}\right)$ (i.e., $q$ value). 
Table 3. Enrichment of disease and disorder sub-categories in nonpleiotropic and pleiotropic gene sets.

$\boldsymbol{P}_{\text {Fisher }}$ is $p$ value from the Fisher test; $\boldsymbol{P}_{\text {FDR }}$ is FDR adjusted $p$ value.

Score is IPA score defined as sum of minus log-baseten transformed FDR adjusted $p$ values.

\begin{tabular}{|c|c|c|c|c|c|}
\hline \multirow[b]{2}{*}{ Disease and disorder sub-category } & \multicolumn{2}{|c|}{ Non-pleiotropic set } & \multicolumn{2}{|c|}{ Pleiotropic set } & \multirow[b]{2}{*}{ Score } \\
\hline & $P_{\text {Fisher }}$ & $P_{\mathrm{FDR}}$ & $P_{\text {Fisher }}$ & $P_{\mathrm{FDR}}$ & \\
\hline Crohn disease & $3.32 \mathrm{E}-06$ & $1.52 \mathrm{E}-04$ & $1.20 \mathrm{E}-03$ & $3.34 \mathrm{E}-02$ & 5.29 \\
\hline Inflammatory bowel disease & $9.32 \mathrm{E}-05$ & $2.43 \mathrm{E}-03$ & $9.08 \mathrm{E}-04$ & $3.17 \mathrm{E}-02$ & 4.11 \\
\hline Familial Alzheimer's disease & $6.69 \mathrm{E}-04$ & $1.25 \mathrm{E}-02$ & $1.01 \mathrm{E}-02$ & $5.82 \mathrm{E}-02$ & 3.14 \\
\hline Familial encephalopathy & $6.89 \mathrm{E}-04$ & $1.27 \mathrm{E}-02$ & $1.09 \mathrm{E}-03$ & $3.34 \mathrm{E}-02$ & 3.37 \\
\hline Syndromic encephalopathy & 7.17E-04 & $1.31 \mathrm{E}-02$ & $5.10 \mathrm{E}-03$ & $4.62 \mathrm{E}-02$ & 3.22 \\
\hline Familial schizophrenia & $2.55 \mathrm{E}-03$ & $3.41 \mathrm{E}-02$ & $4.85 \mathrm{E}-03$ & $4.46 \mathrm{E}-02$ & 2.82 \\
\hline Hypoplasia & $3.59 \mathrm{E}-03$ & $4.32 \mathrm{E}-02$ & 4.24E-03 & 4.11E-02 & 2.75 \\
\hline Chronic inflammatory disorder & $4.60 \mathrm{E}-03$ & $5.03 \mathrm{E}-02$ & $4.23 \mathrm{E}-03$ & 4.11E-02 & 2.68 \\
\hline
\end{tabular}

Table 4. Top 20 disease and disorder sub-categories enriched either in the non-pleiotropic or pleiotropic gene set.

\begin{tabular}{|c|c|c|c|c|c|}
\hline \multicolumn{3}{|l|}{ Non-pleiotropic set } & \multicolumn{3}{|l|}{ Pleiotropic set } \\
\hline Disease/disorder sub-category & $P_{\text {Fisher }}$ & $P_{\mathrm{FDR}}$ & Disease/disorder sub-category & $P_{\text {Fisher }}$ & $P_{\mathrm{FDR}}$ \\
\hline Cerebral disorder & $2.11 \mathrm{E}-10$ & $2.75 \mathrm{E}-08$ & Progressive neuropathy & $1.00 \mathrm{E}-07$ & $1.23 \mathrm{E}-04$ \\
\hline Late-onset Alzheimer's disease & 4.17E-09 & $3.41 \mathrm{E}-07$ & Progressive neuromuscular disease & $5.22 \mathrm{E}-08$ & $1.23 \mathrm{E}-04$ \\
\hline Cognitive impairment & $1.77 \mathrm{E}-05$ & $6.08 \mathrm{E}-04$ & Neuromuscular disease & $2.21 \mathrm{E}-07$ & $1.81 \mathrm{E}-04$ \\
\hline Autism or intellectual disability & $3.18 \mathrm{E}-05$ & $9.93 \mathrm{E}-04$ & Progressive motor neuropathy & $5.28 \mathrm{E}-07$ & $2.58 \mathrm{E}-04$ \\
\hline Congenital malformation of brain & $3.27 \mathrm{E}-05$ & $1.00 \mathrm{E}-03$ & Multiple sclerosis & $9.44 \mathrm{E}-07$ & $3.20 \mathrm{E}-04$ \\
\hline Congenital neurological disorder & $3.54 \mathrm{E}-05$ & $1.07 \mathrm{E}-03$ & Progressive neurological disorder & $6.77 \mathrm{E}-06$ & $1.20 \mathrm{E}-03$ \\
\hline Mental retardation & $5.21 \mathrm{E}-05$ & $1.49 \mathrm{E}-03$ & Disorder of basal ganglia & $1.37 \mathrm{E}-05$ & $1.97 \mathrm{E}-03$ \\
\hline Cockayne syndrome type I & $6.83 \mathrm{E}-05$ & $1.87 \mathrm{E}-03$ & Progressive parkinsonism & $1.86 \mathrm{E}-05$ & $2.40 \mathrm{E}-03$ \\
\hline $\begin{array}{l}\text { Autism spectrum disorder or intel- } \\
\text { lectual disability }\end{array}$ & $8.44 \mathrm{E}-05$ & $2.24 \mathrm{E}-03$ & Susceptibility to multiple sclerosis & $2.93 \mathrm{E}-05$ & 2.99E-03 \\
\hline Nonspecific mental retardation & $2.36 \mathrm{E}-04$ & $5.53 \mathrm{E}-03$ & Movement disorders & $2.73 \mathrm{E}-05$ & 2.99E-03 \\
\hline Dysgenesis & $2.61 \mathrm{E}-04$ & $5.93 \mathrm{E}-03$ & Parkinson's disease & $1.26 \mathrm{E}-04$ & $9.92 \mathrm{E}-03$ \\
\hline Amyloidosis & $3.98 \mathrm{E}-04$ & 8.37E-03 & Creutzfeldt-Jakob disease & $1.46 \mathrm{E}-04$ & $1.03 \mathrm{E}-02$ \\
\hline Aplasia or hypoplasia & 4.17E-04 & $8.61 \mathrm{E}-03$ & Hypersomnia & $1.46 \mathrm{E}-04$ & $1.03 \mathrm{E}-02$ \\
\hline Alzheimer disease & 4.57E-04 & $9.19 \mathrm{E}-03$ & Insulin-dependent diabetes mellitus & $1.47 \mathrm{E}-04$ & $1.03 \mathrm{E}-02$ \\
\hline Microangiopathy & $6.16 \mathrm{E}-04$ & $1.19 \mathrm{E}-02$ & Cirrhosis of liver & $1.64 \mathrm{E}-04$ & $1.12 \mathrm{E}-02$ \\
\hline Aplasia & $6.32 \mathrm{E}-04$ & $1.22 \mathrm{E}-02$ & Severe Alzheimer's disease & $2.04 \mathrm{E}-04$ & $1.24 \mathrm{E}-02$ \\
\hline Early-onset obesity & $6.68 \mathrm{E}-04$ & $1.25 \mathrm{E}-02$ & Systemic lupus erythematosus & 2.37E-04 & $1.42 \mathrm{E}-02$ \\
\hline Lesioning of aorta & $6.72 \mathrm{E}-04$ & $1.25 \mathrm{E}-02$ & Contact dermatitis or eczema & $2.83 \mathrm{E}-04$ & $1.61 \mathrm{E}-02$ \\
\hline Abnormality of cerebral cortex & $7.60 \mathrm{E}-04$ & $1.38 \mathrm{E}-02$ & Severe COVID-19 & $3.13 \mathrm{E}-04$ & $1.74 \mathrm{E}-02$ \\
\hline Non-ketotic hyperglycinemia & $1.00 \mathrm{E}-03$ & $1.73 \mathrm{E}-02$ & Primary biliary cirrhosis & $3.93 \mathrm{E}-04$ & $1.96 \mathrm{E}-02$ \\
\hline
\end{tabular}

$\boldsymbol{P}_{\text {Fisher }}$ is $p$ value from the Fisher test; $\boldsymbol{P}_{\mathbf{F D R}}$ is false discovery rate adjusted $p$ value.

was characterized by sleep disorder (hypersomnia) and specific autoimmune conditions, e.g., insulindependent diabetes mellitus, systemic lupus erythematosus, primary biliary cirrhosis, and rheumatoid arthritis. Enrichment of the immune-mediated conditions was mainly driven by the HLA locus genes.

\section{Biological functions}

The analysis of biological functions showed that tissue development was the only common category in the top five categories for the non-pleiotropic and pleiotropic gene sets (Figure 1C and 1D). Top 
categories of biological functions highlighted nervous system development for the non-pleiotropic gene set and cellular assembly and organization linked to cellular function and maintenance for the pleiotropic one.

Comparative analysis of biological functions in the pleiotropic (Supplementary Table S4) and nonpleiotropic (Supplementary Table S5) gene sets identified 20 common functional annotations related to brain development and synapse formation, organization of the cytoskeleton, organismal death, spatial learning, and nest-building behavior (Table 5).

Top characteristic sub-categories for the pleiotropic gene set included specific microtubule-related terms (e.g., quantity of microtubules), formation of senile plaques, specific behaviors (e.g., grooming, emotional behaviors), early gonadogenesis (e.g., elongation of gonadal cell lines), immune-related processes, heavy metal and water transport, synaptic events, etc. (Table 6). Apart from cell proliferation of tumor cell lines $\left(p_{\mathrm{FDR}}=8.36 \times 10^{-5}\right)($ Supplementary Table S5), top specific terms for the non-pleiotropic gene set were related to the development of the brain and neuronal morphology, cell viability and neuronal cell death, and the process of transcription (Table 6).

\section{Discussion}

Here, we comprehensively examined pleiotropic predisposition to AD and EDU using Fisher's method and omnibus test applied to summary statistics reported in large-scale univariate GWAS of AD [19] and EDU [20]. Fisher's method performs pleiotropic meta-analysis by combining probabilities of $\mathrm{AD}$ and EDU for carriers of the risk variants, and, therefore, it disregards correlation between phenotypes. The omnibus test adjusts the estimates for such correlation. Accordingly, Fisher's method can be considered as a reference, and comparative analysis of the results using this method and the omnibus test helps in evaluating the role of correlation between $\mathrm{AD}$ and EDU.

Meta-analysis of pleiotropic associations for SNPs that did not attain GW significance in the univariate GWAS of EDU or AD $\left(5 \times 10^{-8}<p<\right.$ 0.1 ) identified 53 SNPs for which pleotropic significance achieved GW level using Fisher's method
Table 5 Biological functions enriched in both the non-pleiotropic and pleiotropic gene sets.

$\boldsymbol{P}_{\text {Fisher }}$ is $p$ value from the Fisher test; $\boldsymbol{P}_{\text {FDR }}$ is FDR adjusted $p$ value.

Score is IPA score defined as sum of minus log-baseten transformed FDR adjusted $p$ values.

\begin{tabular}{|c|c|c|c|c|c|}
\hline \multirow[b]{2}{*}{ Biological functions } & \multicolumn{2}{|c|}{ Non-pleiotropic set } & \multicolumn{2}{|c|}{ Pleiotropic set } & \multirow[b]{2}{*}{ Score } \\
\hline & $P_{\text {Fisher }}$ & $P_{\mathrm{FDR}}$ & $P_{\text {Fisher }}$ & $P_{\mathrm{FDR}}$ & \\
\hline Development of CNS & $1.68 \mathrm{E}-09$ & $1.50 \mathrm{E}-07$ & $1.50 \mathrm{E}-02$ & $6.95 \mathrm{E}-02$ & 7.98 \\
\hline Development of neurons & $2.18 \mathrm{E}-08$ & $1.66 \mathrm{E}-06$ & $6.26 \mathrm{E}-04$ & $2.54 \mathrm{E}-02$ & 7.38 \\
\hline Microtubule dynamics & 7.07E-07 & $4.18 \mathrm{E}-05$ & $3.44 \mathrm{E}-04$ & $1.87 \mathrm{E}-02$ & 6.11 \\
\hline Organization of cytoplasm & $5.85 \mathrm{E}-07$ & $3.54 \mathrm{E}-05$ & $7.18 \mathrm{E}-04$ & $2.74 \mathrm{E}-02$ & 6.01 \\
\hline Morphogenesis of neurons & 4.85E-07 & $3.01 \mathrm{E}-05$ & $3.30 \mathrm{E}-03$ & $3.40 \mathrm{E}-02$ & 5.99 \\
\hline Formation of cellular protrusions & $3.09 \mathrm{E}-07$ & $1.99 \mathrm{E}-05$ & $7.58 \mathrm{E}-03$ & $5.35 \mathrm{E}-02$ & 5.97 \\
\hline Organization of cytoskeleton & $1.79 \mathrm{E}-06$ & $9.02 \mathrm{E}-05$ & $6.46 \mathrm{E}-04$ & $2.55 \mathrm{E}-02$ & 5.64 \\
\hline Neuritogenesis & $1.38 \mathrm{E}-06$ & $7.23 \mathrm{E}-05$ & $1.03 \mathrm{E}-02$ & $5.91 \mathrm{E}-02$ & 5.37 \\
\hline Guidance of axons & $2.62 \mathrm{E}-05$ & 8.48E-04 & $6.31 \mathrm{E}-03$ & 4.62E-02 & 4.41 \\
\hline Spatial learning & $5.80 \mathrm{E}-05$ & $1.64 \mathrm{E}-03$ & $8.73 \mathrm{E}-03$ & $5.51 \mathrm{E}-02$ & 4.04 \\
\hline Organismal death & $1.31 \mathrm{E}-04$ & $3.25 \mathrm{E}-03$ & $9.21 \mathrm{E}-04$ & $3.17 \mathrm{E}-02$ & 3.99 \\
\hline Excitatory postsynaptic potential & $1.49 \mathrm{E}-04$ & $3.67 \mathrm{E}-03$ & $2.68 \mathrm{E}-03$ & $3.34 \mathrm{E}-02$ & 3.91 \\
\hline Neurotransmission & $5.93 \mathrm{E}-04$ & $1.15 \mathrm{E}-02$ & $3.08 \mathrm{E}-03$ & $3.34 \mathrm{E}-02$ & 3.42 \\
\hline Development of CNS cells & $7.63 \mathrm{E}-04$ & $1.38 \mathrm{E}-02$ & 7.64E-04 & $2.87 \mathrm{E}-02$ & 3.40 \\
\hline Development of head & $3.47 \mathrm{E}-04$ & $7.54 \mathrm{E}-03$ & $1.14 \mathrm{E}-02$ & $6.48 \mathrm{E}-02$ & 3.31 \\
\hline Nest building behavior & $1.93 \mathrm{E}-03$ & $2.85 \mathrm{E}-02$ & $4.02 \mathrm{E}-03$ & $3.96 \mathrm{E}-02$ & 2.95 \\
\hline Quantity of filaments & $2.50 \mathrm{E}-03$ & $3.40 \mathrm{E}-02$ & $1.80 \mathrm{E}-03$ & $3.34 \mathrm{E}-02$ & 2.94 \\
\hline Development of cerebral cortex & $2.59 \mathrm{E}-03$ & $3.45 \mathrm{E}-02$ & $5.33 \mathrm{E}-03$ & $4.62 \mathrm{E}-02$ & 2.80 \\
\hline Quantity of cells & $4.48 \mathrm{E}-03$ & 4.93E-02 & $2.72 \mathrm{E}-03$ & 3.34E-02 & 1.48 \\
\hline Developmental process of synapse & $3.81 \mathrm{E}-03$ & 4.47E-02 & $1.52 \mathrm{E}-02$ & $6.95 \mathrm{E}-02$ & 1.16 \\
\hline
\end{tabular}


Table 6. Top 25 biological functions enriched either in the non-pleiotropic or pleiotropic gene set.

\begin{tabular}{|c|c|c|c|c|c|}
\hline \multicolumn{3}{|l|}{ Non-pleiotropic set } & \multicolumn{3}{|l|}{ Pleiotropic set } \\
\hline Biological functions & $P_{\text {Fisher }}$ & $P_{\mathrm{FDR}}$ & Biological functions & $P_{\text {Fisher }}$ & $P_{\mathrm{FDR}}$ \\
\hline Formation of brain & $2.20 \mathrm{E}-06$ & 1.07E-04 & Quantity of microtubules & $9.29 \mathrm{E}-05$ & 7.84E-03 \\
\hline Cell movement of neurons & 7.03E-06 & 2.81E-04 & Formation of senile plaques & $9.73 \mathrm{E}-05$ & 7.94E-03 \\
\hline Morphology of nervous tissue & 7.93E-06 & $3.15 \mathrm{E}-04$ & Dissociation of microtubules & $1.46 \mathrm{E}-04$ & $1.03 \mathrm{E}-02$ \\
\hline Differentiation of neuroglia & 8.78E-06 & $3.44 \mathrm{E}-04$ & Grooming & $1.81 \mathrm{E}-04$ & $1.20 \mathrm{E}-02$ \\
\hline Cell viability & $1.33 \mathrm{E}-05$ & 4.89E-04 & Elongation of gonadal cell lines & $2.04 \mathrm{E}-04$ & $1.24 \mathrm{E}-02$ \\
\hline Morphology of nervous system & $1.46 \mathrm{E}-05$ & $5.25 \mathrm{E}-04$ & Emotional behavior & $2.65 \mathrm{E}-04$ & $1.55 \mathrm{E}-02$ \\
\hline Transcription & $1.52 \mathrm{E}-05$ & $5.46 \mathrm{E}-04$ & Lack of CD4+ T-lymphocytes & 4.34E-04 & $2.08 \mathrm{E}-02$ \\
\hline Cell viability of neurons & $1.58 \mathrm{E}-05$ & $5.61 \mathrm{E}-04$ & Differentiation of natural T-regulatory cells & $4.34 \mathrm{E}-04$ & $2.08 \mathrm{E}-02$ \\
\hline Shape change of neurons & $1.59 \mathrm{E}-05$ & $5.61 \mathrm{E}-04$ & $\begin{array}{l}\text { Hydrolysis of phosphatidylinositol phos- } \\
\text { phate }\end{array}$ & $5.29 \mathrm{E}-04$ & $2.23 \mathrm{E}-02$ \\
\hline $\begin{array}{l}\text { Abnormal morphology of cerebral neo- } \\
\text { cortex }\end{array}$ & $1.61 \mathrm{E}-05$ & $5.63 \mathrm{E}-04$ & Instability of microtubules & $6.33 \mathrm{E}-04$ & $2.54 \mathrm{E}-02$ \\
\hline Abnormal morphology of commissure & $1.70 \mathrm{E}-05$ & $5.88 \mathrm{E}-04$ & Morphology of thymus gland & 9.87E-04 & $3.34 \mathrm{E}-02$ \\
\hline Morphology of neurons & $1.83 \mathrm{E}-05$ & $6.21 \mathrm{E}-04$ & Beat of cells & $1.14 \mathrm{E}-03$ & $3.34 \mathrm{E}-02$ \\
\hline Differentiation of nervous system & $1.87 \mathrm{E}-05$ & $6.30 \mathrm{E}-04$ & Export of heavy metal & $1.14 \mathrm{E}-03$ & 3.34E-02 \\
\hline Cell survival & $1.91 \mathrm{E}-05$ & $6.36 \mathrm{E}-04$ & Uptake of water & $1.14 \mathrm{E}-03$ & 3.34E-02 \\
\hline Morphology of somatosensory cortex & $1.91 \mathrm{E}-05$ & $6.36 \mathrm{E}-04$ & Function of synapse & $1.29 \mathrm{E}-03$ & $3.34 \mathrm{E}-02$ \\
\hline Migration of neurons & $2.34 \mathrm{E}-05$ & 7.68E-04 & Homeostasis of leukocytes & $1.67 \mathrm{E}-03$ & 3.34E-02 \\
\hline Formation of rhombencephalon & $2.84 \mathrm{E}-05$ & 8.98E-04 & Abnormal morphology of thymus gland & $1.72 \mathrm{E}-03$ & $3.34 \mathrm{E}-02$ \\
\hline Survival of cerebellar cortex cells & $2.98 \mathrm{E}-05$ & $9.34 \mathrm{E}-04$ & Abnormal morphology of iris & $1.79 \mathrm{E}-03$ & $3.34 \mathrm{E}-02$ \\
\hline Abnormal morphology of forebrain & $3.23 \mathrm{E}-05$ & $1.00 \mathrm{E}-03$ & Fear conditioning & $1.79 \mathrm{E}-03$ & 3.34E-02 \\
\hline Neuronal cell death & $3.64 \mathrm{E}-05$ & $1.09 \mathrm{E}-03$ & Quantity of amacrine cells & $1.79 \mathrm{E}-03$ & $3.34 \mathrm{E}-02$ \\
\hline Abnormal morphology of brain & $3.87 \mathrm{E}-05$ & $1.15 \mathrm{E}-03$ & Abnormal morphology of lens fiber cells & $2.37 \mathrm{E}-03$ & $3.34 \mathrm{E}-02$ \\
\hline Branching of neurites & $4.03 \mathrm{E}-05$ & $1.19 \mathrm{E}-03$ & Anxiety-like behavior & $2.38 \mathrm{E}-03$ & $3.34 \mathrm{E}-02$ \\
\hline Collapse of growth cone & $4.05 \mathrm{E}-05$ & $1.19 \mathrm{E}-03$ & Development of body axis & $2.44 \mathrm{E}-03$ & $3.34 \mathrm{E}-02$ \\
\hline Necrosis & 4.07E-05 & $1.19 \mathrm{E}-03$ & Release of neurotransmitter & $2.48 \mathrm{E}-03$ & $3.34 \mathrm{E}-02$ \\
\hline Development of metencephalon & 4.27E-05 & $1.24 \mathrm{E}-03$ & $\begin{array}{l}\text { Differentiation of regulatory T lympho- } \\
\text { cytes }\end{array}$ & $2.72 \mathrm{E}-03$ & $3.34 \mathrm{E}-02$ \\
\hline
\end{tabular}

$\boldsymbol{P}_{\text {Fisher }}$ is $p$ value from the Fisher test; $\boldsymbol{P}_{\mathbf{F D R}}$ is false discovery rate adjusted $p$ value.

or the omnibus test. The vast majority of them, 46 of 53 SNPs (86.8\%), showed pleiotropic signals using Fisher's method, but only 10 of these 46 SNPs attained pleiotropic GW significance. A commonly anticipated role of correlation between phenotypes is that it attenuates pleiotropic association because of overlapped signals due to either biological pleiotropy (influence of one genetic variant on more than one trait [29]) or mediated pleiotropy (indirect association with a downstream phenotype through an intermediate phenotype [29]). Previously, we showed that correlation between phenotypes might not necessarily attenuate pleiotropic signals but can also strengthen them [18] (see also
"Pleiotropic meta-analysis" section in Methods). Despite counter-intuitiveness, this phenomenon, called antagonistic genetic heterogeneity, appears to be common in the genetics of complex (heterogeneous) traits because genetic predisposition to them may not be driven by the same mechanism as their correlation [17]. Here, we show that the antagonistic genetic heterogeneity increases the significance of the pleiotropic associations for 51 SNPs, making all of them attain the GW level, and suggests additional pleiotropic associations. Thus, contrary to the common expectation, Fisher's method may provide more conservative estimates of pleiotropy than the omnibus test. 
In contrast, Fisher's method identified pleiotropic associations only for 25 of 206 SNPs (12.1\%), which attained the GW significance in prior GWAS of EDU or AD. Also, the antagonistic heterogeneity was less pronounced in this set of SNPs affecting eight of 25 pleiotropic SNPs.

Larger fractions of pleiotropic associations and antagonistic heterogeneity for SNPs, which did not attain GW significances in the univariate GWAS of $\mathrm{AD}$ and $\mathrm{EDU}$ compared to those which attained such significances, support a role of heterogeneity of genetic architecture of $\mathrm{AD}$ and EDU driven by different biological mechanisms. Our functional enrichment analysis of genes harboring pleiotropic and nonpleiotropic SNPs shows both common and specific mechanisms associated with diseases and bio-functions for these two sets.

The non-pleiotropic and pleiotropic gene sets were characterized by enrichment for cancers at different sites (although this enrichment was more pronounced in the non-pleiotropic gene set), some familial brain disorders, including familial AD, and Crohn's disease (Figures 1A and 1B and Table 3). Studies report that chronic inflammation may be connected to neurodegeneration and increase cancer risk [30, 31]. For example, chronic inflammatory conditions of the digestive system increase the risk of developing gastrointestinal malignancies [32]. Patients with inflammatory bowel disease showed positive association with dementia including AD and Parkinson's disease (PD) $[33,34]$. Prior studies reported genetic overlap between $\mathrm{AD}$ and immune-mediated diseases including Crohn's disease [35].

Common bio-functions in the pleiotropic and nonpleiotropic gene sets were related to the development of the brain and synapse formation, organization of the cytoskeleton, organismal death, spatial learning, and nest-building behavior (Table 5). Processes related to the organization of the cytoskeleton (e.g., microtubule dynamics) play roles during the development of neuronal morphology, synapse formation and maintenance, and neurodegeneration [36]. Spatial learning is necessary for orientation in space and the formation of episodic memories, which is the most severely affected aspect of memory in $\mathrm{AD}$ [37]. AD has been characterized by spatial disorientation presented from its early stages and degradation with disease progression [38, 39]. Nest building is related to social behavior and characterizes activities of daily living, which are impaired in people affected by progressive neurodegenerative disorders such as AD and PD [40]. Impairment in nest building is a characteristic feature of multiple transgenic mouse AD models [41]. Also, children with neurodevelopmental disorders usually have poorer activities of daily living performance [42, 43].

In addition to enrichment of multiple cancer types, the non-pleiotropic gene set was characterized by gene enrichment in age-associated cognitive impairment and late-onset $\mathrm{AD}$, along with neurodevelopmental disorders, including congenital encephalopathy, ASD, and mental retardation (Table 4 and Supplementary Table S4). Both cancer and neurodegenerative diseases such as late-onset $\mathrm{AD}$ are common in aging, and they may have common underlying age-related mechanisms. Epidemiological studies show, however, that the relationship between these diseases is complex [44-47]. Enrichment of cancers is in line with the close relationship between cancer and developmental biology. Indeed, embryonic development is characterized by synergistic regulation when cells become organized in functional structures, whereas cancer is characterized by dysregulation of well-behaved structures, i.e., by disorder [48, 49]. It might be hypothesized that dysregulation of the transcriptional programs during embryonal development causes abnormal morphology and developmental disorders and leads to dysregulation in latelife, causing tumorigenesis and cancer progression. Neurodevelopmental disorders can lead to behavioral and learning problems and visual and hearing impairments and directly affect mental processes by limiting educational opportunities and affecting academic achievements.

Analysis of bio-functions for the non-pleiotropic gene set highlighted morphological development of the nervous system, cell survival and death, and transcription process (Table 6 and Supplementary Table S4). Terms related to the morphology of the nervous system indicate anomalies of brain structure and alterations in neuronal morphology, which can contribute to neurodevelopmental disorders and neurodegenerative diseases. For example, a change of the physical structure and the connectivity in the brain was shown in ASD and disorders associated with intellectual disability [50, 51]. Structural changes in specific brain regions and altered neuronal morphology is also characteristic for AD patients [52-54]. 
Neuronal cell viability is important in normal and impaired brain development and during adult neurogenesis. Neuronal cell death is necessary to optimize brain connectivity during development, and it affects cognitive abilities. It is also associated with neurodevelopmental and psychiatric disorders such as schizophrenia and ASD and can occur in neurodegenerative disorders, including AD $[55,56]$. Transcription, the first step of gene expression, highlights the role of transcriptional control in gene regulation. Transcriptional factors are especially important during development. The activity of the transcription factors is linked to multiple neurological disorders and developmental pathologies [57]. Dysregulation of the transcriptional programs is a hallmark of cancer [58].

The pleiotropic gene set was characterized by enrichment of immune and inflammatory pathways driven by HLA genes (Supplementary Table S3). This is in line with enrichment of a number of systemic or organ-specific autoimmune diseases including MS, systemic lupus erythematosus, and type 1 diabetes. In this set, we identified enrichment of neurologic and neuromuscular diseases and movement disorders along with immune-mediated conditions (Table 4 and Supplementary Table S5). A broad spectrum of progressive neurodegenerative diseases affecting various organ systems was enriched in this gene set, including slowly (e.g., MS, PD, severe AD) and rapidly (e.g., Creutzfeldt-Jakob disease) progressive conditions.

Enrichment of bio-functions in the pleiotropic gene set was in line with diseases and disorders characteristic for this set (Table 6 and Supplementary Table S5). For example, enrichment of specific microtubule-related terms (e.g., quantity of microtubules) emphasizes the role of microtubules, which are a highly dynamic part of the cell cytoskeleton playing roles in maintaining neuronal functions and neurodegenerative processes [59]. Microtubules network is a dynamic regulator of skeletal muscle function [60]. Axonal microtubule loss correlates with progressive motor neuron degeneration in motor neuron diseases such as PMN [61]. Microtubule dysfunction is common in many neurodegenerative diseases and movement disorders, including AD and PD [62, 63]. In particular, neurofibrillary tangles, a hallmark of $\mathrm{AD}$, are abnormal accumulations of the microtubule-associated protein tau in a hyperphosphorylated state [63, 64]. Enrichment of specific behaviors such as grooming, emotional behavior, and stress-driven fear- and anxiety-like behavior is consistent with the results from animal models of $\mathrm{AD}$ and PD. For example, progressive degeneration in grooming due to impairments in motor function is considered a symptom of PD and basal ganglia disorders [65, 66]. Emotional symptoms such as stress, anxiety, fear, and depression are common for neurodegenerative diseases, including AD, PD, and MS [67-69]. Our functional analysis highlights the role of $\mathrm{T}$ lymphocytes in immune regulation and links the immune system with neurological disorders (including $\mathrm{AD}$ and $\mathrm{MS}$ ) and the set-specific autoimmune diseases [70-72]. Phosphoinositides signaling (e.g., hydrolysis of phosphatidylinositol phosphate) has several critical roles in the nervous system, including synaptic transmission and cytoskeletal function in neurons [73]. Phosphatidylinositol levels are also affected in AD [74]. Heavy metal toxicity (e.g., export of heavy metal) has been linked to neurological problems and progressive neurological disorders, including AD, PD, and MS [75]. In addition, microtubules play important roles in lens fiber cell functions (e.g., abnormal morphology of lens fiber cells) [76]. Vision problems are the source of behavioral impairments, which impact learning opportunities affecting thus cognition.

Despite the rigor of this study, we acknowledge its limitations. The first is the use of summary statistics rather than individual-level data. Summary statistics limit the ability to accurately model the role of correlation between $\mathrm{AD}$ and EDU in pleiotropic predisposition to these phenotypes because this correlation was not reported in those studies. This limitation was partly offset by estimating the phenotypic correlation between $\mathrm{AD}$ and EDU from three independent studies available to us and the genetic correlation between them evaluated from the reported summary statistics. The second is the lack of replication statistics from independent GWAS. This limitation is partly offset by leveraging the results of large-scale GWAS. The third is that different number of genes in the pleiotropic and non-pleiotropic sets (75 vs. 195) may affects our comparative functional analysis.

Thus, leveraging summary statistics from two large-scale univariate GWAS of $\mathrm{AD}$ and EDU, we identified pleiotropic associations of SNPs from multiple loci with both these phenotypes. We showed that a large fraction of the pleiotropic associations was affected by a counterintuitive phenomenon of antagonistic genetic heterogeneity, which appeared to 
be widespread in the genetics of complex traits. Our comparative functional enrichment analysis of pleiotropic and non-pleiotropic gene sets identified potential differences in biological mechanisms of AD and EDU. The non-pleiotropic gene set was mainly characterized by late-onset AD along with neurodevelopmental disorders and processes related to brain development and neuronal morphology. The pleiotropic gene set was associated with processes characteristic for a broad spectrum of progressive neuromuscular and neurologic diseases and immune-mediated conditions.

Author contribution A.M.K. conceived and designed the study and wrote the paper, E.L. prepared data for the analysis, Y.L. coded statistical tests and performed statistical analyses, I.C. performed functional enrichment analysis. E.L., Y.L., and I.C. contributed to writing the paper.

Funding This research was supported by Grants R01 AG061853, R01 AG065477, and R01 AG070488 from the National Institute on Aging. The funders had no role in study design, data collection, and analysis, decision to publish, or preparation of the manuscript. The content is solely the authors' responsibility and does not necessarily represent the official views of the National Institutes of Health. Correlation coefficients were evaluated using data obtained through $\mathrm{dbGaP}$ (accession numbers phs000007.v31 [FHS], phs000287.v7 [CHS], phs000168.v2 [LOADFS]). See extended acknowledgment in the Supplementary Acknowledgement file.

Data availability The data are publicly available from published GWAS.

Code availability Not applicable

Declarations

Ethics approval Not applicable

Consent to participate Not applicable

Consent for publication Not applicable

Conflict of interest The authors declare no competing interests.

\section{References}

1. Rocca WA, et al. Trends in the incidence and prevalence of Alzheimer's disease, dementia, and cognitive impairment in the United States. Alzheimers Dement. 2011;7:80-93. https://doi.org/10.1016/j.jalz.2010.11. 002.

2. 2021 Alzheimer's disease facts and figures (2021) Alzheimers dement 17:327-406. 10.1002/alz.12328

3. Gao S, Burney HN, Callahan CM, Purnell CE, Hendrie HC. Incidence of dementia and alzheimer disease over time: a meta-analysis. J Am Geriatr Soc. 2019;67:1361-9. https://doi.org/10.1111/jgs.16027.

4. Larson EB, Yaffe K, Langa KM. New insights into the dementia epidemic. N Engl J Med. 2013;369:2275-7. https://doi.org/10.1056/NEJMp1311405.

5. Matthews KA, Xu W, Gaglioti AH, Holt JB, Croft JB, Mack D, McGuire LC. Racial and ethnic estimates of Alzheimer's disease and related dementias in the United States $(2015-2060)$ in adults aged $>/=65$ years. Alzheimers Dement. 2019;15:17-24. https://doi.org/10.1016/j. jalz.2018.06.3063.

6. Livingston $\mathrm{G}$, et al. Dementia prevention, intervention, and care: 2020 report of the Lancet Commission. Lancet. 2020;396:413-46. https://doi.org/10.1016/S01406736(20)30367-6.

7. Sando SB, et al. Risk-reducing effect of education in Alzheimer's disease. Int J Geriatr Psychiatry. 2008;23:115662. https://doi.org/10.1002/gps.2043.

8. Stern Y. Cognitive reserve in ageing and Alzheimer's disease. Lancet Neurol. 2012;11:1006-12. https://doi.org/10. 1016/S1474-4422(12)70191-6.

9. Fratiglioni L, Wang HX. Brain reserve hypothesis in dementia. J Alzheimers Dis. 2007;12:11-22. https://doi. org/10.3233/jad-2007-12103.

10. Katzman R, et al. Clinical, pathological, and neurochemical changes in dementia: a subgroup with preserved mental status and numerous neocortical plaques. Ann Neurol. 1988;23:138-44. https://doi.org/10.1002/ana.410230206.

11. Stern Y (2006) Cognitive reserve and Alzheimer disease Alzheimer disease and associated disorders. 20:112-117. https://doi.org/10.1097/01.wad.0000213815.20177.19

12. Stern Y, et al. Brain networks associated with cognitive reserve in healthy young and old adults. Cereb Cortex. 2005;15:394-402. https://doi.org/10.1093/cercor/bhh142.

13. Bocancea DI, van Loenhoud AC, Groot C, Barkhof F, van der Flier WM, Ossenkoppele R. Measuring resilience and resistance in aging and Alzheimer disease using residual methods: a systematic review and meta-analysis. Neurology. 2021;97:474-88. https://doi.org/10.1212/WNL. 0000000000012499.

14. Mitnitski AB, Mogilner AJ, Rockwood K. Accumulation of deficits as a proxy measure of aging. Sci World J. 2001;1:323-36.

15. Canevelli M, et al. Biomarkers and phenotypic expression in Alzheimer's disease: exploring the contribution of frailty in the Alzheimer's disease neuroimaging initiative. GeroScience. 2021;43:1039-51. https://doi.org/10.1007/ s11357-020-00293-y.

16. Wallace LMK, et al. Neuropathologic burden and the degree of frailty in relation to global cognition and dementia. Neurology. 2020;95:e3269-79. https://doi.org/ 10.1212/WNL.0000000000010944.

17. Kulminski AM, et al. Strong impact of natural-selectionfree heterogeneity in genetics of age-related phenotypes. 
Aging (Albany NY). 2018;10:492-514. https://doi.org/10. 18632/aging.101407.

18. Kulminski AM, et al. Pleiotropic meta-analysis of agerelated phenotypes addressing evolutionary uncertainty in their molecular mechanisms. Front Genet. 2019;10:433. https://doi.org/10.3389/fgene.2019.00433.

19. Lambert JC, et al. Meta-analysis of 74,046 individuals identifies 11 new susceptibility loci for Alzheimer's disease. Nat Genet. 2013;45:1452-8. https://doi.org/10.1038/ ng. 2802 .

20. Okbay A, et al. Genome-wide association study identifies 74 loci associated with educational attainment. Nature. 2016;533:539-42. https://doi.org/10.1038/nature17671.

21. Fisher RAS (1970) Statistical methods for research workers. 14th ed. edn. Oliver and Boyd, Edinburgh

22. Bolormaa $\mathrm{S}$, et al. A multi-trait, meta-analysis for detecting pleiotropic polymorphisms for stature, fatness and reproduction in beef cattle. PLoS Genet. 2014;10:e1004198. https://doi.org/10.1371/journal.pgen. 1004198.

23. Xu X, Tian L, Wei LJ. Combining dependent tests for linkage or association across multiple phenotypic traits. Biostatistics. 2003;4:223-9. https://doi.org/10.1093/biost atistics/4.2.223

24. Zhu X, et al. Meta-analysis of correlated traits via summary statistics from GWASs with an application in hypertension. Am J Hum Genet. 2015;96:21-36. https://doi.org/ 10.1016/j.ajhg.2014.11.011.

25. Cupples LA, Heard-Costa N, Lee M, Atwood LD (2009) Genetics analysis workshop 16 problem 2: the Framingham. Heart Study data BMC Proc 3 Suppl 7:S3

26. Fried LP, et al. The Cardiovascular Health Study: design and rationale. Ann Epidemiol. 1991;1:263-76.

27. Lee JH, Cheng R, Graff-Radford N, Foroud T, Mayeux R, National Institute on Aging Late-Onset Alzheimer's Disease Family Study G. Analyses of the National Institute on aging late-onset Alzheimer's disease family study: implication of additional loci. Arch Neurol. 2008;65:1518-26. https://doi.org/10.1001/archneur.65.11.1518.

28. Benjamini Y, Hochberg Y. Controlling the false discovery rate - a practical and powerful approach to multiple testing. J Roy Stat Soc B Met. 1995;57:289-300.

29. Solovieff N, Cotsapas C, Lee PH, Purcell SM, Smoller JW. Pleiotropy in complex traits: challenges and strategies. Nat Rev Genet. 2013;14:483-95. https://doi.org/10. 1038/nrg3461.

30. Coussens LM, Werb Z. Inflammation and cancer. Nature. 2002;420:860-7. https://doi.org/10.1038/nature01322.

31. Stephenson J, Nutma E, van der Valk P, Amor S. Inflammation in CNS neurodegenerative diseases. Immunology. 2018;154:204-19. https://doi.org/10.1111/imm.12922.

32. Axelrad JE, Lichtiger S, Yajnik V. Inflammatory bowel disease and cancer: the role of inflammation, immunosuppression, and cancer treatment. World J Gastroenterol. 2016;22:4794-801. https://doi.org/10.3748/wjg.v22.i20. 4794.

33. Brudek T. Inflammatory bowel diseases and Parkinson's disease. J Parkinsons Dis. 2019;9:S331-44. https://doi. org/10.3233/JPD-191729.

34. Zhang B, et al. Inflammatory bowel disease is associated with higher dementia risk: a nationwide longitudinal study. Gut. 2021;70:85-91. https://doi.org/10.1136/ gutjnl-2020-320789.

35. Yokoyama JS, et al. Association between genetic traits for immune-mediated diseases and Alzheimer disease. JAMA Neurol. 2016;73:691-7. https://doi.org/10.1001/ jamaneurol.2016.0150.

36. Munoz-Lasso DC, Roma-Mateo C, Pallardo FV, Gonzalez-Cabo P (2020) Much more than a scaffold: cytoskeletal proteins in neurological disorders. Cells 9. https:// doi.org/10.3390/cells9020358

37. Tromp D, Dufour A, Lithfous S, Pebayle T, Despres O. Episodic memory in normal aging and Alzheimer disease: Insights from imaging and behavioral studies. Ageing Res Rev. 2015;24:232-62. https://doi.org/10. 1016/j.arr.2015.08.006.

38. Giuliani F, Schenk F. Vision, spatial cognition and intellectual disability. Res Dev Disabil. 2015;37:202-8. https://doi.org/10.1016/j.ridd.2014.11.015.

39. Vlcek K, Laczo J. Neural correlates of spatial navigation changes in mild cognitive impairment and Alzheimer's disease. Front Behav Neurosci. 2014;8:89. https:// doi.org/10.3389/fnbeh.2014.00089.

40. Galasko D, Schmitt F, Thomas R, Jin S, Bennett D, Alzheimer's disease cooperative S. Detailed assessment of activities of daily living in moderate to severe Alzheimer's disease. J Int Neuropsychol Soc. 2005;11:446-53. https://doi.org/10.1017/s1355617705050502.

41. Jirkof P. Burrowing and nest building behavior as indicators of well-being in mice. J Neurosci Methods. 2014;234:139-46. https://doi.org/10.1016/j.jneumeth. 2014.02.001.

42. Bal VH, Kim SH, Cheong D, Lord C. Daily living skills in individuals with autism spectrum disorder from 2 to 21 years of age. Autism. 2015;19:774-84. https://doi. org/10.1177/1362361315575840.

43. Kottorp A, Bernspång B, Fisher AGJAOTJ (2003) Activities of daily living in persons with intellectual disability: strengths and limitations in specific motor and process skills 50:195-204

44. Driver JA, et al. Inverse association between cancer and Alzheimer's disease: results from the Framingham Heart Study. Brit Med J. 2012;344:e1442. https://doi.org/10. 1136/bmj.e1442.

45. Ganguli M (2015) Cancer and Dementia: It's Complicated Alzheimer disease and associated disorders 29:177-182. https://doi.org/10.1097/WAD.0000000000 000086

46. Houck AL, Seddighi S, Driver JA. At the Crossroads between neurodegeneration and cancer: a review of overlapping biology and its implications. Curr Aging Sci. 2018;11:77-89. https://doi.org/10.2174/1874609811 666180223154436.

47. Ospina-Romero M, et al. Association between Alzheimer Disease and cancer with evaluation of study biases: a systematic review and meta-analysis. JAMA Netw Open. 2020;3:e2025515. https://doi.org/10.1001/jamanetwor kopen.2020.25515.

48. Aiello NM, Stanger BZ. Echoes of the embryo: using the developmental biology toolkit to study cancer. Dis Model Mech. 2016;9:105-14. https://doi.org/10.1242/dmm. 023184 . 
49. Ma Y, Zhang P, Wang F, Yang J, Yang Z, Qin H. The relationship between early embryo development and tumourigenesis. J Cell Mol Med. 2010;14:2697-701. https://doi. org/10.1111/j.1582-4934.2010.01191.x.

50. Dierssen M, Ramakers GJ. Dendritic pathology in mental retardation: from molecular genetics to neurobiology. Genes Brain Behav. 2006;5(Suppl 2):48-60. https://doi. org/10.1111/j.1601-183X.2006.00224.x.

51. Hashem S, et al. Genetics of structural and functional brain changes in autism spectrum disorder. Transl Psychiatry. 2020;10:229. https://doi.org/10.1038/ s41398-020-00921-3.

52. Ghatak S et al. (2019) Mechanisms of hyperexcitability in Alzheimer's disease hiPSC-derived neurons and cerebral organoids vs isogenic controls. eLife 8. https://doi.org/10. 7554/eLife.50333

53. Grutzendler J, Helmin K, Tsai J, Gan WB. Various dendritic abnormalities are associated with fibrillar amyloid deposits in Alzheimer's disease. Ann N Y Acad Sci. 2007;1097:30-9. https://doi.org/10.1196/annals.1379.003.

54. Ohnishi T, Matsuda H, Tabira T, Asada T, Uno M. Changes in brain morphology in Alzheimer disease and normal aging: is Alzheimer disease an exaggerated aging process? AJNR Am J Neuroradiol. 2001;22:1680-5.

55. Mavroudis I, Petridis F, Kazis D, Njau SN, Costa V, Baloyannis SJ. Purkinje cells pathology in Alzheimer's disease. Am J Alzheimers Dis Other Dement. 2019;34:439-49. https://doi.org/10.1177/1533317519 859200 .

56. Pfisterer U, Khodosevich K. Neuronal survival in the brain: neuron type-specific mechanisms. Cell Death Dis. 2017;8:e2643. https://doi.org/10.1038/cddis.2017.64.

57. Santiago C, Bashaw GJ (2014) Transcription factors and effectors that regulate neuronal morphology. Development (Cambridge, England) 141:4667-4680. https://doi.org/10. 1242/dev.110817

58. Bradner JE, Hnisz D, Young RA. Transcriptional addiction in cancer. Cell. 2017;168:629-43. https://doi.org/10. 1016/j.cell.2016.12.013.

59. Lasser M, Tiber J, Lowery LA. The role of the microtubule cytoskeleton in neurodevelopmental disorders. Front Cell Neurosci. 2018;12:165. https://doi.org/10.3389/fncel. 2018.00165.

60. Sebastien M, et al. Deletion of the microtubule-associated protein 6 (MAP6) results in skeletal muscle dysfunction. Skelet Muscle. 2018;8:30. https://doi.org/10.1186/ s13395-018-0176-8.

61. Schaefer MK, Schmalbruch H, Buhler E, Lopez C, Martin N, Guenet JL, Haase G. Progressive motor neuronopathy: a critical role of the tubulin chaperone TBCE in axonal tubulin routing from the Golgi apparatus. J Neurosci. 2007;27:8779-89. https://doi.org/10.1523/JNEUROSCI. 1599-07.2007.

62. Pellegrini L, Wetzel A, Granno S, Heaton G, Harvey $\mathrm{K}$. Back to the tubule: microtubule dynamics in Parkinson's disease. Cellular and molecular life sciences : CMLS. 2017;74:409-34. https://doi.org/10.1007/ s00018-016-2351-6.

63. Sferra A, Nicita F, Bertini E. Microtubule dysfunction: a common feature of neurodegenerative diseases. Int J Mol Sci. 2020;21. https://doi.org/10.3390/ijms21197354.
64. Venkatramani A, Panda D. Regulation of neuronal microtubule dynamics by tau: implications for tauopathies. Int J Biol Macromol. 2019;133:473-83. https://doi.org/10. 1016/j.ijbiomac.2019.04.120.

65. Kalueff AV, Stewart AM, Song C, Berridge KC, Graybiel AM, Fentress JC. Neurobiology of rodent self-grooming and its value for translational neuroscience. Nat Rev Neurosci. 2016;17:45-59. https://doi.org/10.1038/nrn.2015.8.

66. Pelosi A, Girault JA, Herve D. Unilateral lesion of dopamine neurons induces grooming asymmetry in the mouse. PLoS One. 2015;10:e137185. https://doi.org/10.1371/ journal.pone.0137185.

67. Askari F, Ghajarzadeh M, Mohammadifar M, Azimi A, Sahraian MA, Owji M. Anxiety in patients with multiple sclerosis: association with disability, depression, disease type and sex. Acta Med Iran. 2014;52:889-92.

68. Broen MP, Narayen NE, Kuijf ML, Dissanayaka NN, Leentjens AF. Prevalence of anxiety in Parkinson's disease: a systematic review and meta-analysis. Mov Disord. 2016;31:1125-33. https://doi.org/10.1002/mds.26643.

69. Levenson RW, Sturm VE, Haase CM. Emotional and behavioral symptoms in neurodegenerative disease: a model for studying the neural bases of psychopathology. Annu Rev Clin Psychol. 2014;10:581-606. https://doi.org/ 10.1146/annurev-clinpsy-032813-153653.

70. Ciccocioppo F, et al. The characterization of regulatory T-cell profiles in Alzheimer's disease and Multiple Sclerosis. Sci Rep. 2019;9:8788. https://doi.org/10.1038/ s41598-019-45433-3.

71. Fan KQ, et al. Stress-induced metabolic disorder in peripheral CD4(+) $\mathrm{T}$ cells leads to anxiety-like behavior. Cell. 2019;179:864-79 e819. https://doi.org/10.1016/j. cell.2019.10.001.

72. Lourenco EV, La Cava A. Natural regulatory $\mathrm{T}$ cells in autoimmunity. Autoimmunity. 2011;44:33-42. https://doi. org/10.3109/08916931003782155.

73. Raghu P, Joseph A, Krishnan H, Singh P, Saha S. Phosphoinositides: regulators of nervous system function in health and disease. Front Mol Neurosci. 2019;12:208. https://doi.org/10.3389/fnmol.2019.00208.

74. Desale SE, Chinnathambi S (2021) Phosphoinositides signaling modulates microglial actin remodeling and phagocytosis in Alzheimer's disease Cell communication and signaling : CCS 19:28:https://doi.org/10.1186/ s12964-021-00715-0

75. Chen P, Miah MR, Aschner M (2016) Metals and neurodegeneration. F1000Res 5 10.12688/f1000research.7431.1

76. Logan CM, Menko AS. Microtubules: evolving roles and critical cellular interactions. Exp Biol Med (Maywood). 2019;244:1240-54. https://doi.org/10.1177/1535370219 867296.

Publisher's note Springer Nature remains neutral with regard to jurisdictional claims in published maps and institutional affiliations. 